\title{
A Dynamic Paradigm for Spectrally Efficient Half-Duplex Multi-Antenna Relaying
}

\author{
Young-bin Kim, Student Member, IEEE, Wan Choi, Senior Member, IEEE, \\ Bang Chul Jung, Member, IEEE, and Aria Nosratinia, Fellow, IEEE
}

\begin{abstract}
This paper presents a spectrally efficient protocol for half-duplex multi-relay systems in block fading channels where a direct source-destination link is unavailable. The proposed protocol adaptively selects either successive interference cancelation (SIC) or joint decoding according to the causal decoding status of each relay. We also adopt dynamic refreshing that restarts the protocol whenever it is advantageous to do so, even if the relay decoding set (the set of relays that are able to decode the message) is not empty. The achievable diversity-multiplexing tradeoff (DMT) of the proposed protocol with $m$-antenna nodes is analyzed via a Markov chain whose states are related to the cardinality of a decoding set. This protocol strictly improves the DMT of the existing DF half-duplex relay-selection protocols without decoding delay, and in the low multiplexing gain region is able to meet the DMT upper bound. The main contributions of the paper are the state-dependent decoding strategies in DF multi-relay systems and also the dynamic refresh for the flushing of residual interferences in the system, concepts that may find usefulness beyond the gains in the high-SNR regime.
\end{abstract}

Index Terms-Half-duplex relay, diversity-multiplexing tradeoff, dynamic decoding, dynamic refreshing.

\section{INTRODUCTION}

\section{A. DMT in Half-duplex Relay Networks}

$\mathbf{H}$ ALF-DUPLEX relay protocols were first studied in [1] and analyzed in terms of diversity and multiplexing tradeoff (DMT) which effectively explains a fundamental relationship between spectral efficiency and diversity gain in wireless fading channels [2]. Laneman et al. [1] derived the DMT upper bound of cooperative networks with $K$ halfduplexing relay nodes with a single antenna at each and showed that the DMT upper bound is determined by either a $(K+1) \times 1$ multiple-input-single-output (MISO) channel or a $1 \times(K+1)$ single-input-multiple-output (SIMO) channel if a direct link between source and destination exists, where especially $K=1$.

Although half-duplex operation is more practical than fullduplex operation, it suffers from a multiplexing loss. In

Manuscript received December 25, 2012; revised May 7, 2013; accepted June 24, 2013. The associate editor coordinating the review of this paper and approving it for publication was W. Gerstacker.

This work was supported by the National Research Foundation of Korea (NRF) grant funded by the Korea government "(MEST)" (No. 2011-0013416).

Y.-B. Kim and W. Choi are with the Department of Electrical Engineering, Korea Advanced Institute of Science and Technology (KAIST), Daejeon 305701, Korea (e-mail: aliasing@kaist.ac.kr, wchoi@ee.kaist.ac.kr).

B. C. Jung is with the Dept. of Information and Communication Engineering, Gyeongsang National University, Tongyoung, Korea.

A. Nosratinia is with the the Department of Electrical Engineering, University of Texas at Dallas, TX, U.S.A.

Digital Object Identifier 10.1109/TWC.2013.072613.122053 order to overcome the multiplexing loss in half duplexing amplify-and-forward (AF) and decode-and-forward (DF) relaying strategies, nonorthogonal amplify-and-forward (NAF) and dynamic decode-and-forward (DDF) were proposed. NAF was originally proposed by Nabar et al. [3] and further analyzed by Azarian et al. [4]. NAF attains additional performance gain over simple AF strategy owing to source node's transmission in the second phase (second time slot), but cannot achieve the optimal DMT. DDF was proposed in [4]-[6] independently, and its DMT was analyzed in [4]. In the low multiplexing gain regime, DDF was shown to achieve the optimal DMT for a single relay node with a single antenna. However, in the high multiplexing gain regime (larger than $1 / 2)$, DDF cannot achieve the optimal DMT due to the time spent by relay in listening the signal from source. Furthermore, in the networks with multiple relay nodes, the gap between the optimal DMT and the achievable DMT of NAF or DDF becomes larger as the number of relay nodes increases.

Recently, Yuksel et al. [7] showed that the compressand-forward (CF) strategy achieves the optimal DMT for a single relay node with multiple antennas. In the CF strategy, relay does not decode the received signal, but compresses it under a compression constraint and forwards it to destination. However, in order to define the constraint on compression, $\mathrm{CF}$ requires the relay to perfectly know the full channel state information (CSI) of all channels involved in the transmission. Pawar, et al. [8] proposed quantize-and-map (QM) strategy where the relay does not decode the received signal from source but extracts the most significant bits of the received signal above a noise level by proper quantization and forwards it to destination. QM was shown to achieve the optimal DMT for both a single relay node and multiple half-duplex relay nodes even without full CSI. However, in the network with multiple relay nodes, no interference between relay nodes was assumed. It should also be noted that neither CF nor QM are effective if a direct link between source and destination is absent, because the compressed or quantized signal forwarded by a relay node helps the transmission from source via the direct link.

\section{B. DMT in Half-duplex Multiple Relay Networks}

In multi-relay networks, Escrig [9] proposed on-demand cooperation with relay selection (OXIDE) initiated by destination in view of medium access control (MAC) layer. The OXIDE protocol is a combination of incremental relaying and opportunistic SDF in the multi-relay environment with a direct 
path. With the help of additional dimensions provided by retransmissions, the OXIDE protocol compensates for the halfduplex loss and achieves optimal DMT ${ }^{1}$. Without additional dimensions by retransmission like $\mathrm{H}-\mathrm{ARQ}$, one may also address the loss with transmission strategies where the source transmits in every transmission interval concurrently with alternating relay transmissions.

For the DF strategy, [11] and [12] proposed alternate relaying protocols for the network with two half-duplex relay nodes without a direct link and with a direct link, respectively. In [11], one relay node forwards the received signal from source and the other relay node decodes the signal from a source by dealing with the inter-relay interference as a Gaussian noise. In [12], one relay node forwards the received signal from a source and the other relay node receives the signal from the source by using successive interference cancelation (SIC) that decodes the interference signal from the other relay first if the interference is strong enough or decodes the desired signal by dealing with the interference as a Gaussian noise. This protocol can achieve the upper bound of DMT if the number of phases is sufficiently large and the channel between relay nodes is good enough to guarantee the success of SIC at relay nodes. However, the success of SIC is not always guaranteed in general. Bletsas and Dimitriou [13] evaluated opportunistic relaying with the successive transmission like [11] and [12] in the presence of a thermal noise as well as interference from a selected single relay in the multiple-relay environment. No inter-relay interference was taken into account in the analysis of outage probability of the proposed protocol by assuming directional antennas at relay nodes. No consideration of interrelay interference makes the proposed method neither general nor practical as the authors mentioned in their paper.

Using AF strategy, Yang and Belfiore [14] proposed a sequential slotted amplify-and-forward (SSAF) technique with multiple half-duplex relay nodes. In the SSAF scheme, a pair of relay nodes forwards the amplified version of the received signal which was received in the previous phase in every phase while other relays listen the signal from a source. The SSAF scheme achieves the optimal DMT when the interference between relay nodes does not exist. In [15], a random sequence (RS) scheme was proposed with multiple half-duplex AF relay nodes when a direct link between a source node and a destination node does not exist. RS allows successive transmissions at a node by selecting proper multihop paths. The RS scheme was proved to achieve the DMT of the $K \times 1$ MISO upper bound as the number of blocks increase in a quasi-static channel where channel state is not varied over one block consisting of multiple slots. A few features of the RS method include the assumption of a quasi-static channel with very long coherence interval (many transmission blocks), fundamental dependence on the linearity of the AF protocol, and simultaneous decoding of several message blocks at the destination with an associated decoding delay. In contrast, this paper addresses the DF protocol, does not assume very long coherence intervals, and the decoding occurs within each transmission block which produces a relatively shorter

\footnotetext{
${ }^{1}$ However, because delay caused by retransmissions is not reflected in DMT, some studies define a new DMT measure such as diversity-multiplexing-delay tradeoff (DMDT) [10] for fair comparison.
}

decoding delay. Recently, [16] showed that the half duplex loss can be recovered by coded cooperation and successive cancelation at relays in DF relay networks allowing one time protocol re-initiation. As the number of assisting relays increases, the proposed multi-relay coded cooperation protocol in [16] achieves the DMT upper bound.

Although some DMT optimal strategies have been known, they suffer from decoding delay which makes the strategies practically infeasible. To the best of our knowledge, in multirelay DF networks without a direct source-destination link, existing methods fall short of the DMT upper bound. Some DMT-optimal strategies exist either in the presence of a direct link or with AF strategies that allow long codewords going across multiple blocks. For example, the method of [15] is limited to $\mathrm{AF}$ and requires knowledge of an equivalent channel matrix whose size increases proportionally to $\Theta\left(B^{2} K^{2}\right)$, where $B$ is the number of repetitions of the multihop paths and $K$ is the number of relays. From a perspective of practical implementation, the encoding/decoding delays and the burden on the channel estimation in the scheme of [15] is considerable. In order to improve the DMT performance for DF strategies in systems without a direct link, Tannious and Nosratinia [17] proposed a spectrally efficient relaying scheme called multi-hop relay selection (MHRS) that combines joint decoding and SIC in a multiple relay network without a direct link. In this scheme, a codeword is transmitted at the source node in every phase. At relay nodes, the received signal from a source node in the previous phase is utilized to cancel out interference from the relay node which is selected to forward the received data to a destination node. The MHRS scheme cannot achieve the optimal DMT bound. The emphasis of this paper is improvement of the DF protocol in multi-relay channels without a direct link. The present work provides several important improvements over [17] which are outlined in the following subsection.

\section{Contributions}

We consider the DF half-duplex multi-relay scenario without a direct source-destination link, and propose a protocol whose assumptions on inter-relay interference and the required number of phases are more relaxed than several previous works [11]-[20]. We propose several new techniques and are able to strictly improve the DMT performance, in other words, reduce the so-called half-duplex loss. Our contributions are summarized as follows:

- Introducing dynamical joint decoding and SIC at relay nodes, based on the decoding status of the relay node in the previous phase. The use of joint decoding and SIC in the context of cognitive radio [17] has so far been static, in the sense that at each multiplexing gain, [17] decides to use SIC or joint decoding once and for all, regardless of the instantaneous state of the system. In this work, if a relay node succeeds in decoding the received data of the previous phase, it performs SIC. If not, it performs joint decoding. We analyze the outage probability of the proposed protocol via a new analytical framework based on the Markov chain in a steady state. Our analysis in addition further generalizes [17] by allowing multiple antennas on all nodes. 
- We also introduce dynamic refreshing of the proposed protocol. Dynamic refreshing enables a restart of the transmission protocol even if the decoding set is not empty in order to enhance diversity gain in the moderate and high multiplexing gain regime.

- The proposed protocol improves the DMT of existing DF half-duplex relay-selection systems, and specifically in low multiplexing gains achieves the DMT upper bound. However, we emphasize that the contributions of this paper are mainly the introduction of dynamic (statedependent) decoding and refresh strategies in the DF half-duplex relay selection systems, concepts that may find usefulness beyond just the gains in the high-SNR regime.

\section{Organization and Notations}

The rest of this paper is organized as follows. In Section II, we present system and channel models of the proposed protocol. The description of our proposed protocol is given in Section III. DMT analysis using a Markov chain are provided in Section IV. In Section V, we introduce dynamic refreshing of the proposed protocol and analyze its DMT. In the Section VI, we briefly show that how much the proposed protocol can be improved when CSI at the transmitter (CSIT) only for joint decoding is used. Numerical results are given in Section VII and finally, conclusions are drawn in Section VIII.

Throughout this paper, as used in [2], $f(\rho) \doteq \rho^{v}$ if

$$
\lim _{\rho \rightarrow \infty} \frac{\log (f(\rho))}{\log \rho}=v .
$$

$a^{+}$denotes $\max (0, a)$ for any real value $a$, and $\log (\cdot)$ denotes the base-2 logarithm. [a,b] denotes a set,

$\{x \in \mathbb{R} \mid a \leq x \leq b\}$, where $\mathbb{R}$ is the set of real values. We denote conjugate transpose of the matrix $\mathbf{A}$ as $\mathbf{A}^{\dagger}$ and its determinant as $|\mathbf{A}|$. $\mathbf{I}_{M}$ denotes a $M \times M$ identity matrix.

\section{System And Channel Models}

We consider a decode-and-forward (DF) based cooperative wireless communication system utilizing a multi-phase halfduplex relaying protocol, as shown in Fig. 1. The system consists of a single source node, a single destination node, and $K$ relay nodes denoted by $s, d$, and $r \in\{1, \cdots, K\}$, respectively. Each has $m$ antennas. It is assumed that a direct link between the source and destination nodes does not exist due to the geometry of relay networks as assumed in [11], [13], and [17]. The number of whole phases is $n$.

Assuming a relay set is denoted as $\mathcal{S}_{\text {relay }}=\{1, \cdots, K\}$, the received signal at node $B$ from node $A$ in the $i$ th phase is given by

$$
\mathbf{y}_{B}=\mathbf{H}_{A, B}^{(i)} \mathbf{x}_{A}^{(i)}+\mathbf{n}_{B}^{(i)}
$$

where $\mathbf{x}_{A}^{(i)} \in \mathcal{C}^{m \times 1}$ indicates the signal transmitted from node $A$ in the $i$ th phase and $\mathbf{H}_{A, B}^{(i)} \in \mathcal{C}^{m \times m}$ represents the fading channel from node $A$ to node $B$ in the $i$ th phase. The terms $A$ and $B$ can be either the source $(s)$ and the relay $\left(r_{j}, \forall r_{j} \in\right.$ $\left.\mathcal{S}_{\text {relay }}\right)$ or the relay and the destination $(d)$, respectively. The channel fading coefficients, $h_{j k}, j, k=1, \ldots, m$ are assumed to be a complex Gaussian random variable $\sim \mathcal{C N}(0,1)$. The
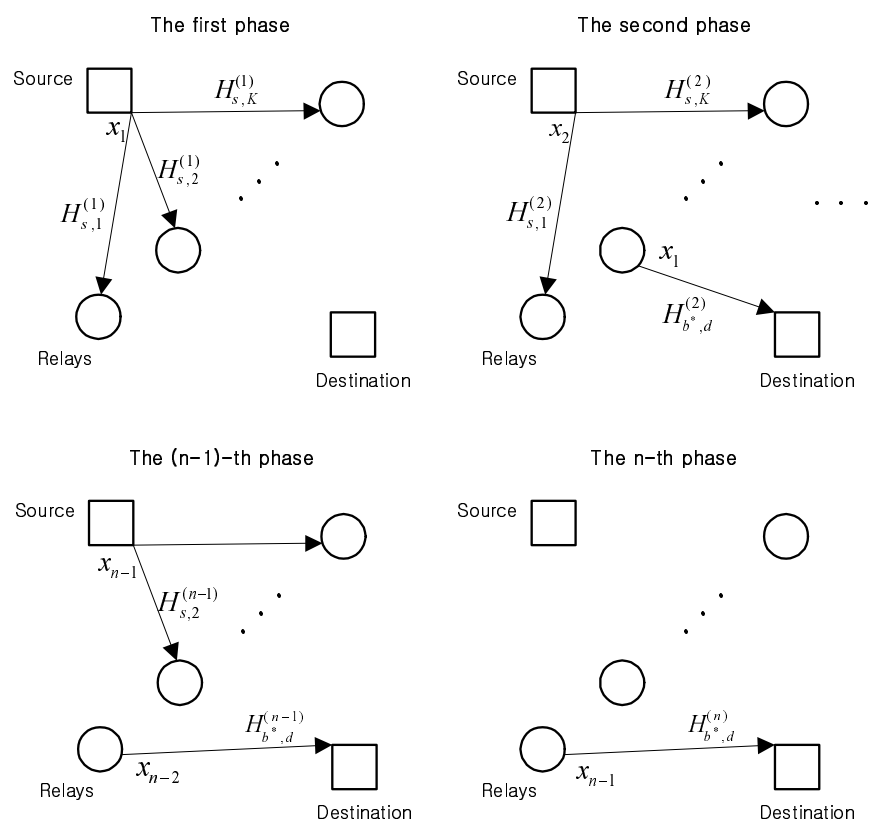

Fig. 1. System model.

channels between two nodes are assumed to be frequency-flat and vary independently in every phase. In this paper, perfect CSI at the receiver (CSIR) is assumed. Transmissions are assumed to be synchronized. Pilot training sequences are used for channel estimation at the receiver, and it is assumed that channel estimation errors are not occurred. In our protocol, all phases have the same time length and the fixed time duration for channel estimation in each phase is assumed to be negligible compared to data transmission duration in each phase. Especially, the source-to-relay channel and interrelay channel (between the selected relay and the other relays) can be estimated at once by broadcasted orthogonal pilots from the source and the selected relay, respectively. Pilots are assumed to be orthogonal in time, i.e., sent one after each other in successive time intervals without overlap. Because we are doing DMT and the number of relays does not increase with SNR, still the amount of time spent on pilots is negligible. Relay-to-destination channel can also be estimated simultaneously by using orthogonal pilots. Pilots are assumed to be orthogonal in time, i.e., sent one after each other in successive time intervals without overlap. Because the key metric is DMT and the number of relays does not increase with SNR, still the amount of time spent on pilots is negligible. ${ }^{2}$ An additive white Gaussian noise (AWGN) at node $B$ in the $i$ th phase is denoted by $\mathbf{n}_{B}^{(i)}$ and it is assumed to follow a complex Gaussian distribution $\sim \mathcal{C N}\left(0, N_{0}\right)$.

For simplicity, all nodes are assumed to have the same transmit power constraint $P$, and SNR at each receiver is denoted $\rho$, i.e., $\rho=\frac{P}{N_{0}}$. Let $\mathcal{D}^{(i)}$ be a decoding set consisting of the relay nodes that successfully decode the transmitted

\footnotetext{
${ }^{2}$ Throughout this paper, pilot signaling is just for the support of CSIR in a multi-relay environment generally assumed in many works [1] and resource handling for the signaling in higher layers including MAC (medium access control) layer is beyond the scope of this paper.
} 
data from the source node in the $i$ th phase and $\left|\mathcal{D}^{(i)}\right|$ be the cardinality of the decoding set.

In this paper, the source node is assumed to transmit an independent packet consisting of a codeword obtained from random Gaussian codebook in every phase. The length of a codeword in a phase is assumed to be sufficiently large so that the error event is dominated by the outage event but spans only one coherence time. In the first phase, the source node transmits (broadcasts) a packet to relay nodes. Then, in the second phase, the relay node having the largest relay-to-destination channel gain in the decoding set $\left(\mathcal{D}^{(1)}\right)$ is selected. The index of the relay selected in the second phase is determined by $b^{*(2)}=\arg \max _{r \in \mathcal{D}^{(1)}}\left\|\mathbf{H}_{r, d}^{(2)}\right\|_{F}^{2}$, where $\|\cdot\|_{F}$ is the Frobenius norm of a matrix.

This relay selection can be executed in the centralized manner by the destination as in [17] and [21]. Under the CSIR assumption, the destination can indicate the selected relay by forwarding a small amount of feedback bits after recognizing which relay's channel is the best. In the proposed protocol, each relay informs its status to the destination whether it has successfully decoded the received signal using a single feedback bit. Then, the destination broadcasts to all relays, and one of the relays is selected using $\log K$ bits. Therefore, $1+\frac{\log K}{K}$ bit per node per phase is required for relay selection. Meanwhile, at the same phase (the second phase), the source node transmits another packet to the relay nodes. After the second phase, the selected relay node forwards the packet received in the previous phase, and the source node broadcasts the next packet in every phase. In the last phase $(i=n)$, the selected relay node forwards the packet received in the previous phase while the source node is silent. The channel from the selected relay to other relay nodes is assumed to be known to the other relay nodes through channel estimation.

\section{Proposed Spectrally EfFicient Cooperative RElaying PRotocol With DyNAMIC REFRESHING}

In most phases (except for $i=1, n$ ), all relays except for the selected relay suffer from interference from the selected relay when the relays decode the received packet from the source. As for the relay nodes which are not selected and belong to the decoding set, they can decode the received packet in the current phase without interference caused by the selected relay using the SIC technique because they already successfully decoded the packet of the previous phase and knew the channel from the selected relay. Let $\mathcal{E}_{S I C}$ be the set of outage events in the $i$-th phase when SIC is performed at the $j$-th relay node. Then, $\mathcal{E}_{S I C}$ can be represented as

$$
\mathcal{E}_{S I C}=\left\{\log \left|\mathbf{I}_{M}+\rho \mathbf{H}_{s, r_{j}}^{(i)} \mathbf{H}_{s, r_{j}}^{(i) \dagger}\right|<R\right\},
$$

where $R$ is the given rate.

When the relays that are not in the decoding set decode the received packet in the current phase, they try to perform joint decoding which means concurrent decoding of interference and signal at each relay (as opposed to successive cancelation, which decodes the interference first). In each phase, relay nodes except for the selected relay have a multiple-access channel from the source (desired packet) and the selected relay (interference). For successful decoding by joint decoding, a rate pair of the desired signal and interference should be within the multiple-access channel capacity region or the desired signal can be decoded under interference. Let $\mathcal{E}_{J D}$ be the set of outage events with joint decoding in the $i$-th phase for the $j$-th relay. Then, $\mathcal{E}_{J D}$ can be expressed as intersections of the following events like a Z-channel [23]:

$$
\mathcal{E}_{1}=\left\{\begin{array}{c}
\log \left|\mathbf{I}_{M}+\rho \mathbf{H}_{s, r_{j}}^{(i)} \mathbf{H}_{s, r_{j}}^{(i) \dagger}\right|<R \quad \cup \\
\log \left|\mathbf{I}_{M}+\rho \mathbf{H}_{b^{*}, r_{j}}^{(i)} \mathbf{H}_{b^{*}, r_{j}}^{(i)}\right|<R \quad \cup \\
\log \left|\mathbf{I}_{M}+\rho \mathbf{H}_{s, r_{j}}^{(i)} \mathbf{H}_{s, r_{j}}^{(i) \dagger}+\rho \mathbf{H}_{b^{*}, r_{j}}^{(i)} \mathbf{H}_{b^{*}, r_{j}}^{(i) \dagger}\right|<2 R
\end{array}\right\},
$$

$$
\begin{aligned}
\mathcal{E}_{2}= & \left\{\log \mid \mathbf{I}_{M}+\rho \mathbf{H}_{s, r_{j}}^{(i)}\left(\mathbf{I}_{M}+\rho \mathbf{H}_{b^{*}, r_{j}}^{(i) \dagger} \mathbf{H}_{b^{*}, r_{j}}^{(i)}\right)^{-1}\right. \\
& \left.\mathbf{H}_{s, r_{j}}^{(i) \dagger} \mid<R\right\} .
\end{aligned}
$$

From the outage events given by $\mathcal{E}_{J D}$, i.e., $\mathcal{E}_{J D}=\mathcal{E}_{1} \cap \mathcal{E}_{2}$, the condition for successful decoding at the $j$-th relay in the proposed protocol is obtained in Eq. (6) for $2 \leq i \leq n-1$ on the top of the next page.

Note that one of the main differences of the proposed protocol from [17] is dynamic decoding. While the selection of decoding strategy in [17] is determined by DMT performance according to multiplexing gain, that in the proposed scheme is determined by the state of the relay whether it is in the decoding set or not. Note also that the number of phases in [17] is restricted less than the number of relays nodes in order to prevent zero diversity gain because the relay nodes excluded from the decoding set are not likely to contribute to the overall diversity gain any longer. The restriction on the number of transmission phases hurts the generality of analysis and does not match with the ultimate objective of a spectrally efficient protocol that asymptotically recovers multiplexing gain to $m$.

Without the restriction on the number of transmission phases, in order to optimize the refreshing cycle, we consider dynamic refreshing in the proposed protocol which is another main difference from [17]. The main idea of dynamic refreshing is to allow refreshing and restarting of the proposed protocol even before the decoding set is empty. Dynamic refreshing secures diversity gain even in the high multiplexing gain regime. Let $N_{c}$ be the cardinality of the decoding set which determines the time to restart the transmission protocol. For example, $N_{c}=2$ means that the protocol restarts when the cardinality of the decoding set is less than three.

\section{Diversity Multiplex Tradeoff Analysis with MARKOV CHAIN}

In this section, we analyze outage probability and DMT of the proposed protocol for a sufficiently large number of $n$ phases. For DMT analysis, note that multiplexing gain and diversity gain are defined, respectively, as

$$
r=\lim _{\rho \rightarrow \infty} \frac{\log R(\rho)}{\log \rho}, \quad d=\lim _{\rho \rightarrow \infty}-\frac{\log \mathrm{P}_{\text {out }}(\rho)}{\log \rho}
$$

where $R(\rho)$ represents the transmission rate, and $\mathrm{P}_{\text {out }}$ denotes outage probability. In order to compute outage probability in the $i$-th phase, we should consider the $(i-1)$ outage 


$$
r_{j} \in \mathcal{D}^{(i)} \text { if }\left\{\begin{array}{cc}
\log \left|\mathbf{I}_{M}+\rho \mathbf{H}_{s, r_{j}}^{(i)} \mathbf{H}_{s, r_{j}}^{(i) \dagger}\right| \geq R, & \text { for }\left|\mathcal{D}^{(i-1)}\right|=0, \\
\log \left|\mathbf{I}_{M}+\rho \mathbf{H}_{s, r_{j}}^{(i)} \mathbf{H}_{s, r_{j}}^{(i) \dagger}\right| \geq R, & \text { for }\left|\mathcal{D}^{(i-1)}\right| \neq 0 \text { and } r_{j} \in \mathcal{D}^{(i-1)} \\
\mathcal{E}_{J D}^{c}, & \text { for }\left|\mathcal{D}^{(i-1)}\right| \neq 0 \text { and } r_{j} \notin \mathcal{D}^{(i-1)}
\end{array}\right.
$$

probabilities in all previous phases to obtain cardinality of the decoding set in the $i$-th phase. However, outage probability in the $i$-th phase follows Markovity, where it depends on only the $(i-1)$-th phase. Let the random variables related to cardinality of the decoding set in $i$-th phase be $\mathbf{D}_{i}$. Outage probability in the $i$-th phase is dependent on all of the previous random variables, i.e., $\left\{\mathbf{D}_{l}\right\}_{l=1}^{i-1}=\left\{\mathbf{D}_{1}, \ldots, \mathbf{D}_{i-1}\right\}$. Owing to the Markovity for $\mathbf{D}$, outage probability in the $i$-th phase denoting $\operatorname{Pr}\left\{\right.$ out $\left._{i}\right\}$ is given by

$$
\begin{aligned}
& \operatorname{Pr}\left\{\text { out }_{i}\right\} \\
& =\sum_{\left\{\mathbf{D}_{l}\right\}_{l=1}^{i=1} \in \mathcal{S}_{\mathcal{D}}} \operatorname{Pr}\left\{\text { out }_{i} \mid\left\{\mathbf{D}_{l}\right\}_{l=1}^{i-1}\right\} \operatorname{Pr}\left\{\left\{\mathbf{D}_{l}\right\}_{l=1}^{i-1}\right\} \\
& =\sum_{\mathbf{D}_{i-1} \in \mathcal{S}_{\mathcal{D}}} \operatorname{Pr}\left\{\text { out }_{i} \mid \mathbf{D}_{i-1}\right\} \operatorname{Pr}\left\{\mathbf{D}_{i-1}\right\} \\
& =\sum_{t=0}^{K} \operatorname{Pr}\left\{\text { out }|| \mathcal{D}^{(i-1)} \mid=t\right\} \operatorname{Pr}\left\{\left|\mathcal{D}^{(i-1)}\right|=t\right\} \\
& =\sum_{t=0}^{K} \operatorname{Pr}\left\{\log \left|\mathbf{I}_{M}+\rho \mathbf{H}_{b^{*}, d}^{(i)} \mathbf{H}_{b^{*}, d}^{(i) \dagger}\right|<R|| \mathcal{D}^{(i-1)} \mid=t\right\} \\
& =\sum_{t=0}^{K}\left[\operatorname{Pr}\left\{\left|\mathcal{D}^{(i-1)}\right|=t\right\}\right.
\end{aligned}
$$

where $\mathcal{S}_{\mathcal{D}}$ is the sample space of random variables on the cardinality of the decoding set. Eq. (8) follows from the Markovity and chain rule and holds due to the assumption that all interference channels are i.i.d., therefore outage probability is independent of the index of the selected relay node. Outage probability in the $i$-th phase is simplified but it is still intractable because outage probability in each phase depends on cardinality of the decoding set in the previous phase. In order to properly handle this complicated system model where $n-1$ codewords are transmitted during $n$ phases, average DMT should be analyzed since DMT in the $i$-th phase depends on both channel state and decoding set cardinality in all previous phases. Thus, we devise a Markov chain for the cardinality of the decoding set so as to reduce analytical complexity and make analysis more precise. Before the Markov chain reaches a steady state, outage probability and DMT in each phase are not static. On the other hand, after the Markov chain arrives at a steady state, outage probability and DMT in each phase become constant for any arbitrary phase. Note that the time until the Markov chain reaches the steady state is negligible in the system model where $n-1$ codewords are transmitted during $n$ phases if $n$ is sufficiently large. This fact can be confirmed by our mathematical analysis in Appendix A.

Define the states of our Markov chain by the cardinality of the decoding set, i.e., $0, \ldots, K$. The transition matrix, $\mathbf{P}$ is defined as

$$
\mathbf{P}=\left(\begin{array}{ccccc}
P_{0,0} & P_{1,0} & \cdots & P_{K-1,0} & P_{K, 0} \\
P_{0,1} & P_{1,1} & \cdots & P_{K-1,1} & P_{K, 1} \\
\vdots & \vdots & \ddots & \vdots & \vdots \\
P_{0, K-1} & P_{1, K-1} & \cdots & P_{K-1, K-1} & P_{K, K-1} \\
P_{0, K} & 0 & \cdots & 0 & 0
\end{array}\right)
$$

where $P_{i, j}$ is the transition probability from state $i$ to $j$. The transition probability $P_{i j}$ from state $i$ to state $j$ is constructed from all possible combinations of SIC and joint decoding. Once dynamic refreshing with $N_{c}$ is adopted, the transition matrix can be formulated as Eq. (13) on the top of the next page. Note that $P_{K-N_{c}, K}=P_{K-N_{c}+1, K}=\cdots=P_{K, K}=0$ in the last row since a selected relay in the decoding set is always forwarding and thus the cardinality of the decoding set cannot be $K$ except for the first phase and the first $N_{c}$ columns are the same since they are transition probabilities from the initial state due to refreshing.

Let $\boldsymbol{\pi}\left(=\left[\pi_{0}, \pi_{1}, \ldots, \pi_{K}\right]^{T}\right)$ be a $(K+1) \times 1$ steady state probability vector for the cardinality of the decoding set and then it is obvious that $\sum_{j=0}^{K} \pi_{j}=1$. After a sufficiently large number of phases, the outage probability can be obtained by using Lemma 1.

Lemma 1: If a Markov chain is irreducible and aperiodic, then either the states are all transient or all states are positive recurrent. If all states of the Markov chain are positive recurrent, the $n$-step transition probability to reach state $j$, $r_{i j}(n)$, converges to a steady state probability $\pi_{j}$ regardless of the initial state $i$. That is,

$$
\boldsymbol{\pi}=\lim _{n \rightarrow \infty} \mathbf{P}_{i}^{n}>0
$$

where $\mathbf{P}$ is a transition matrix of the Markov chain and $\mathbf{P}_{i}^{n}$ is the $i$-th column of the matrix $\mathbf{P}^{n}$. $\boldsymbol{\pi}$ has a unique stationary distribution.

Proof: See the reference [22].

Because the Markov chain reaches a steady state after a sufficient number of phases which is negligible compared to large $n$, the steady state probability, $\pi_{j}, \forall j \in\{0, \cdots, K\}$, can be substituted for the probability of the cardinality of decoding set, $\operatorname{Pr}\left\{\left|\mathcal{D}^{(i-1)}\right|=t\right\}$, after a large number of phases. Correspondingly, the outage probability given in Eq. (11) reduces to

$$
\operatorname{Pr}\left\{\text { out }_{i}\right\}=\sum_{t=0}^{K}\left(\operatorname{Pr}\left\{\log \left|\mathbf{I}_{M}+\rho \mathbf{H}_{r, d}^{(i)} \mathbf{H}_{r, d}^{(i) \dagger}\right|<R\right\}\right)^{t} \cdot \pi_{t} .
$$

The accuracy of the snapshot approach using a Markov chain when the number of phases is not extremely large is verified in Fig. 2 in the next page. The outage probability from Eq. (15) matches well with the outage probability by MonteCarlo simulations. That is, the simulation results validate 


$$
\mathbf{P}=\left(\begin{array}{ccccccc}
P_{0,0} & \cdots & P_{0,0} & P_{K-N_{c}, 0} & \cdots & P_{K-1,0} & P_{K, 0} \\
P_{0,1} & \cdots & P_{0,1} & P_{K-N_{c}, 1} & \cdots & P_{K-1,1} & P_{K, 1} \\
P_{0,2} & \cdots & P_{0,2} & P_{K-N_{c}, 2} & \cdots & P_{K-1,2} & P_{K, 2} \\
\vdots & \cdots & \vdots & \ddots & \vdots & \vdots & \vdots \\
P_{0, K-2} & \cdots & P_{0, K-2} & P_{K-N_{c}, K-2} & \cdots & P_{K-1, K-2} & P_{K, K-2} \\
P_{0, K-1} & \cdots & P_{0, K-1} & P_{K-N_{c}, K-1} & \cdots & P_{K-1, K-1} & P_{K, K-1} \\
P_{0, K} & \cdots & P_{0, K} & 0 & \cdots & 0 & 0
\end{array}\right)
$$

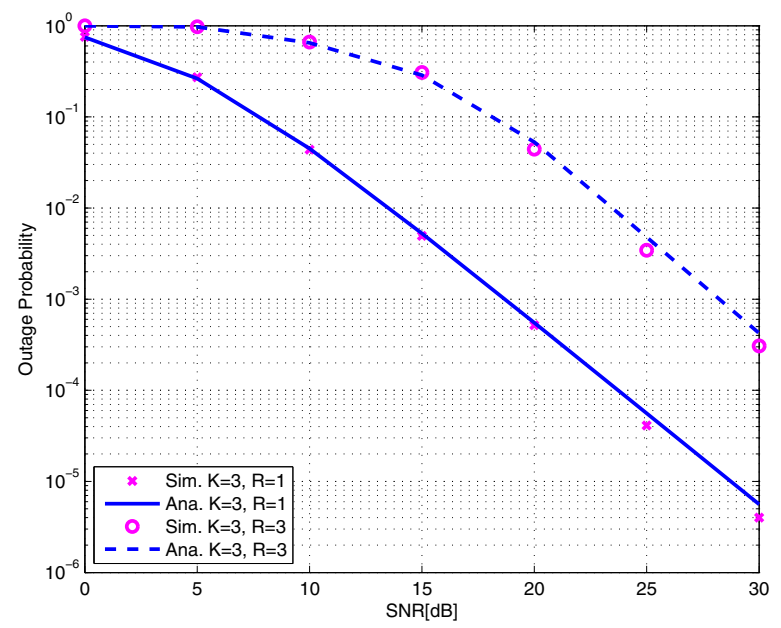

Fig. 2. Comparison simulation of outage probability of the proposed protocol with analysis when $K=3$ and $R=1$ or $R=3$ for 30 phases.

the theoretical analysis using the steady state probability of the decoding set cardinality. Therefore, an arbitrary number of transmission phases can be incorporated in the outage probability analysis using a Markov chain contrary to [17].

Focusing on DMT performance, substitute $R$ with $r \log \rho$ and let

$$
\pi_{t} \doteq \tilde{\pi}_{t}
$$

where $\tilde{\pi}_{t}$ is the dominant scale of $\pi_{t}$. Then, the outage probability scales like

$$
\operatorname{Pr}\left\{\text { out }_{i}\right\} \doteq \sum_{t=0}^{K} \rho^{-t d_{m, m}(r)} \tilde{\pi}_{t},
$$

where $d_{m, m}(r)$ is the optimal $m$ by $m$ MIMO DMT shown in [2], which can be formulated by the piecewise curve, $(m-$ $r)(m-r)^{+}$.

In the proposed protocol, a relay performs SIC if it successfully decodes in the previous phase and if not, joint decoding is performed. In each phase, the signaling overhead in this protocol is the same as that required for MHRS in [17] because each relay node knows its own decoding status. That means no additional overhead is needed in determining a strategy for managing inter-relay interference.

The outage events for SIC and joint decoding are defined in Eq. (3) and Eq. (4), respectively. Therefore, the outage probabilities conditioned on the decoding status in the previous phase scale like

$$
\begin{aligned}
& \operatorname{Pr}\left\{\text { out }_{i} \mid \overline{\text { out }_{i-1}}\right\} \doteq \rho^{-d_{m, m}(r)} \\
& \operatorname{Pr}\left\{\text { out }_{i} \mid \text { out }_{i-1}\right\} \doteq \rho^{-d_{m, m}^{J D}(r)}
\end{aligned}
$$

where $\overline{\text { out }_{i}}$ denotes successful decoding in the $i$ th phase and $d_{m, m}^{J D}(r)$ is DMT of joint decoding in an $m \times m$ MIMO channel. Throughout this paper, the DMT of joint decoding can be obtained from the DMT analysis for a Z-channel [23], [24] because the outage events for joint decoding are the same as those for the Z-channel. DMT of an $m \times m$ MIMO Zchannel without CSI at transmitters (CSIT) is given by [24]

$$
d_{m, m}^{J D}(r)=\min \left\{d_{m, m}(r), d_{m, 2 m}(2 r)\right\} .
$$

DMT of a single-antenna Z-channel is given in Eq. (21) [23] on the top of the next page where $\eta \triangleq \min (1, \beta)$ and $\mu \triangleq \max (1, \beta)$ assuming $\left|\rho \mathbf{H}_{s, r_{j}}^{(i)} \mathbf{H}_{s, r_{j}}^{(i) \dagger}\right|=\rho$ and $\left|\rho \mathbf{H}_{b^{*}, r_{j}}^{(i)} \mathbf{H}_{b^{*}, r_{j}}^{(i) \dagger}\right|=\rho^{\beta}$ on the top of the next page. If $\beta=1$, we can confirm that Eq. (20) is absolutely a super set of Eq. (21), i.e., $d_{1,1}^{J D}(r)=d_{J D}(r, 1)$.

We will focus on the case that the perceived interference power from the selected relay is comparable to the received power of the desired signal, i.e., $\beta=1$.

Theorem 1: When there are $m$-antenna $K$ relays in the absence of a direct link between the source and destination with $m$ antennas at each, the DMT of the upper bound for opportunistic relaying is given by

$$
d_{\text {upper }}(r, m, K)=(K-1) d_{m, m}(r)
$$

Proof: Due to successive forwarding by the selected relay, $K-1$ relays can be candidates for relay selection in every phase. Since all channels are assumed to be i.i.d., $m \times m$ MIMO DMT with $K-1$ selection diversity is the upper bound.

Theorem 2: When each node has $m$ antennas, the DMT of the proposed protocol with dynamic refreshing for $N_{c}$ is given by Eq. (23) on the top of the next page.

Proof: See Appendix A.

Remark 1: When each node has one antenna, the DMT of the proposed protocol with dynamic refreshing for $N_{c}$ is given by Eq. (24) on the top of the next page.

The proposed protocol with dynamic refreshing makes a full recovery from the multiplexing loss caused by half duplex operation and approaches the optimal DMT bound when $0<r \leq \frac{m}{2}$ because the cardinality of the decoding set is asymptotically $K-1$ (i.e., $\rho^{0}$ in the $K$-th element in $\tilde{\boldsymbol{\pi}}$ ) when $0<r \leq \frac{m}{2}$ as shown in Appendix A. Interestingly, the region 


$$
d_{J D}(r, \beta)=\left\{\begin{array}{cc}
\min (1-r, 1+\beta-2(1+\beta) r), & \text { if }(1+\beta) r \leq \eta \\
\mu-(1+\beta) r, & \text { if } \eta<(1+\beta) r \leq \mu
\end{array}\right.
$$

$$
d_{S E}\left(r, m, K, N_{c}\right)=\left\{\begin{array}{ccc}
(K-1) d_{m, m}(r), & \text { if } \quad 0<r \leq \frac{m}{3} \\
\min _{i=1, \cdots, K-1-N_{c}}\left\{(K-1-i) d_{m, m}(r)+\frac{1}{2}\left(i^{2}+i\right) d_{m, m}^{J D}(r)\right\}, & \text { if } & \frac{m}{3}<r \leq \frac{m}{2} \\
N_{c} d_{m, m}\left(\frac{K-N_{c}+1}{K-N_{c}} r\right), & \text { if } & \frac{m}{2}<r \leq m
\end{array}\right.
$$

$$
d_{S E}\left(r, 1, K, N_{c}\right)=\left\{\begin{array}{ccc}
(K-1)(1-r), & \text { if } \quad 0<r \leq \frac{1}{3} \\
\min _{i=1, \cdots, K-1-N_{c}}\left\{K+i^{2}-1-\left(K+2 i^{2}+i-1\right) r\right\}, & \text { if } \quad \frac{1}{3}<r \leq \frac{1}{2} \\
N_{c}\left(1-\frac{K-N_{c}+1}{K-N_{c}} r\right)^{+}, & \text {if } \quad \frac{1}{2}<r \leq 1
\end{array}\right.
$$

where $\frac{m}{3}<r \leq \frac{m}{2}$ is again divided into $K-N_{c}-1$ regions with different DMTs for $1 \leq N_{c} \leq K-2$, and diversity gain grows as $N_{c}$ increases (refer to Appendix A for details). A larger value of $N_{c}$ triggers earlier refreshing that decreases multiplexing gain but improves diversity gain because the cardinality of the decoding set determines diversity gain. When $\frac{m}{2}<r \leq m$, the DMT performance by dynamic refreshing can be optimized by adaptively selecting the value of $N_{c}$ according to multiplexing gain $r$, and multiplexing gain can be $\frac{K-N_{c}+1}{K-N_{c}}$. In conclusion, if multiplexing gain is less than a certain value, dynamic refreshing is not required because the proposed protocol without dynamic refreshing already achieves the DMT upper bound. However, if multiplexing gain is larger than a certain value, adaptive selection of $N_{c}$ according to multiplexing gain significantly improves DMT performance. For example, the optimal value of $N_{c}$ is $K-2$ in the multiplexing gain region where the proposed protocol with dynamic refreshing outperforms the protocol of [17] in terms of DMT. The following corollary identifies the region where dynamic refreshing yields DMT performance gain over the protocol of [17].

Corollary 1: The proposed protocol with dynamic refreshing approximately achieves the upper bound of DMT in the following multiplexing gain regime as $K$ increases and the optimal value of $N_{c}$ is this region becomes $K-2$ :

$$
r_{O P T} \in\left[0, \frac{m}{2}\right]
$$

Proof:

$$
\begin{aligned}
& \left.\lim _{K \rightarrow \infty} d_{S E}\left(r, m, K, N_{c}\right)\right|_{N_{c}=K-2} \\
= & \lim _{K \rightarrow \infty} \max \left\{(K-1) d_{m, m}(r),\right. \\
& \left.(K-2) d_{m, m}(r)+d_{m, m}^{J D}(r)\right\} \\
\stackrel{(a)}{\simeq} & (K-1) d_{m, m}(r) \\
= & d_{\text {upper }}(r, m, K)
\end{aligned}
$$

where $r \in\left[0 \cdot \frac{m}{2}\right]$, and (a) follows from that $(K-1) d_{m, m}(r) \simeq$ $(K-2) d_{m, m}(r)+d_{m, m}^{J D}(r)$ when $K$ goes to infinity.

If dynamic refreshing is applied to the proposed protocol, additional overhead is required for dynamic refreshing. The amount of feedback information depends on the cardinality of the decoding set. The relays in the decoding set send their own status with one bit to the destination node. Then, the destination node knows how many relays are in the decoding set in each phase. If refreshing is needed, the destination node notifies it to relays with one bit.

Assuming perfect feedback, multiplexing gain $r$, and corresponding optimal $N_{c}^{*}$, the required number of feedback bits is equal to

$$
\sum_{k=0}^{K} \operatorname{Pr}(|\mathcal{D}|=k)\left\{K+\operatorname{Pr}\left(k \leq N_{c}^{*}\right)+\operatorname{Pr}\left(k>N_{c}^{*}\right) \log K\right\} .
$$

In particular, since $\operatorname{Pr}(|\mathcal{D}|=K-1)=1$ asymptotically and $N_{c}^{*}=K-2$ when $0 \leq r \leq \frac{1}{2}$ from Theorem 2, the total number of the required feedback bits when $0 \leq r \leq \frac{1}{2}$ is $K+\log K$ bits. Otherwise, when $\frac{1}{2} \leq r \leq 1$, we need the following amount of feedback information in an average sense.

$$
\sum_{k=N_{c}^{*}}^{K} \frac{1}{K-N_{c}^{*}+1}\left\{K+\frac{1}{K-N_{c}^{*}+1}+\frac{\left(K-N_{c}^{*}\right) \log K}{K-N_{c}^{*}+1}\right\}
$$

bits since the steady state probabilities of the states are asymptotically the same - for given $N_{c}^{*}$, there are $K-N_{c}^{*}+1$ states. This result confirms that a finite number of feedback bits per transmit node is sufficient for the proposed protocol ${ }^{3}$.

\section{Numerical Results}

This section compares the DMT performance of the proposed protocol with that of the multi-hop relay selection (MHRS) protocol of [17] which has been known to achieve the best DMT performance until now for the system model without decoding delay which we consider. For example, if $m=1$, the achievable DMT of the MHRS protocol is given by

$$
\begin{aligned}
& d_{M H R S}(r, 1, K, B) \\
& \quad=\max \left\{d_{S C^{\prime}}(r, 1, K, B), d_{J D^{\prime}}(r, 1, K, B)\right\}
\end{aligned}
$$

\footnotetext{
${ }^{3}$ The overhead analysis does not take into account preambles or pilots for packets as [17] did.
} 


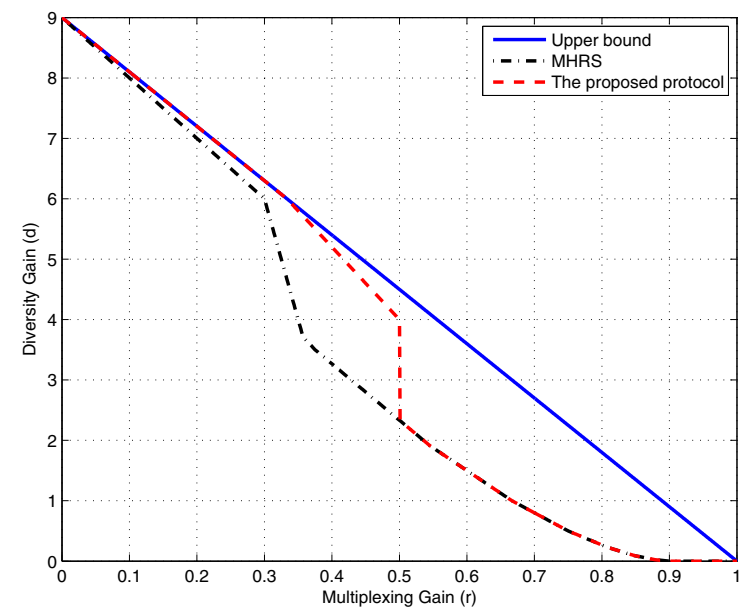

Fig. 3. DMT of the proposed protocol and MHRS scheme case when $K=$ 10 and $m=1$.

where

$$
\begin{gathered}
d_{S C^{\prime}}(r, 1, K, B)=(K-B+1)^{+}\left(1-\frac{B+1}{B} r\right)^{+}, \\
d_{J D^{\prime}}(r, 1, K, B)=\min \left\{(K-1)\left(1-\frac{B+1}{B} r\right)^{+},\right. \\
\left.\min _{t=0, \ldots, K-1}\left[(2 K-2-t)-\frac{B+1}{B} r(4 K-4-3 t)\right]^{+}\right\},
\end{gathered}
$$

and $(B+1)$ is the number of phases until refreshing.

The DMT performance of both schemes largely depends on the time to refresh the protocols; the cardinality of the decoding set determines the refreshing time in the proposed protocol while refreshing occurs after a given number of phases in the MHRS protocol. The number of phases in the MHRS protocol is typically set to be the number of relays in order not to lose diversity gain after a large number of phases. For fair comparison, the decoding set cardinality for refreshing $N_{c}$ and the number of phases are adaptively optimized for both protocols, respectively, and then the achievable DMTs of the protocols are given, respectively, by $\max _{N_{c}} d_{S E}\left(r, 1, K, N_{c}\right)$ and $\max _{n} d_{M H R S}(r, 1, K, B)$ if $m=1$.

Fig. 3 and Fig. 4 show achievable DMT curves of the proposed protocol with dynamic refreshing and the MHRS [17] when $m=1$. When $0<r \leq \frac{1}{3}$, the proposed protocol achieves the DMT upper bound for the given number of relay nodes even without dynamic refreshing. When $\frac{1}{3}<r \leq \frac{1}{2}$, the proposed protocol approaches the DMT upper bound owing to dynamic refreshing and significantly outperforms the MHRS protocol in which joint decoding is switches to SIC beyond $r=0.35$. The optimal value of $N_{c}$ in the proposed protocol with dynamic refreshing is $K-2$ when $\frac{1}{3}<r \leq \frac{1}{2}$. It should be noted that the gap between the DMT upper bound and the proposed protocol does not depend on $K$ and thus the ratio of achievable diversity gains between the proposed protocol and the DMT upper bound for a given multiplexing gain converges to 1 as $K$ increases. While $N_{c}^{*}$ and the multiplexing

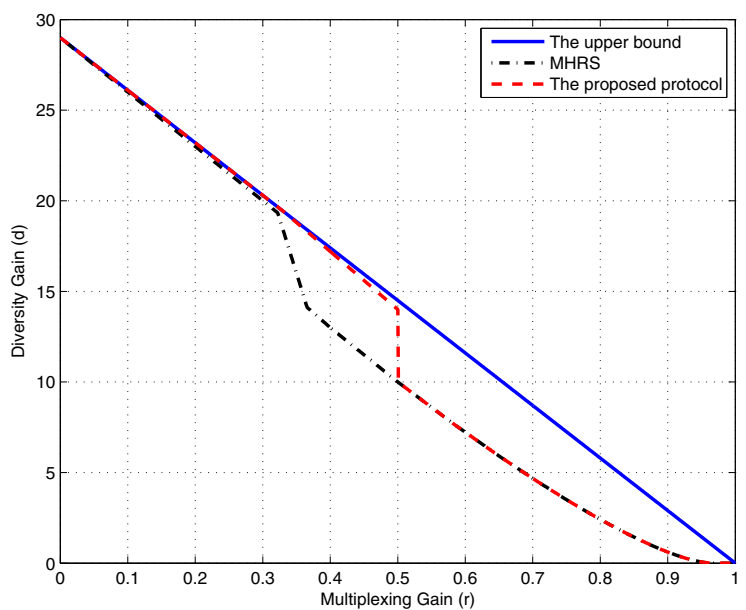

Fig. 4. DMT of the proposed protocol and MHRS scheme when $K=30$ and $m=1$.

loss are fixed when $0<r \leq \frac{1}{2}$, the optimal values of $N_{c}$ and the number of phases vary according to every value of multiplexing gain $r$ when $r>0.5$. Interestingly, both protocols have the same achievable DMT when $r>0.5$ because the proposed protocol always selects SIC in the region while the MHRS already switches from joint decoding to SIC beyond $r=0.35$.

In summary, the proposed protocol with dynamic refreshing achieves the optimal DMT bound in the low multiplexing gain regime and yields the same DMT performance with the MHRS scheme in the relatively high multiplexing gain regime. The gain of the proposed protocol comes form dynamic refreshing based on the decoding set cardinality and joint consideration of joint decoding and SIC. However, SIC is always used for both protocols in the relatively high multiplexing gain regime given by $r_{S I C} \in\left[\frac{m}{2}, m\right]$.

\section{CONCLUSION}

We proposed a spectral efficient protocol with multiple half duplex relay nodes in the absence of a direct link from source to destination using dynamic refreshing if all nodes have $m$ antennas at each. We analyzed its DMT by using a Markov chain in steady states to reflect the cardinality of the decoding set. Our analysis is applicable to arbitrary number of transmission phases contrary to existing studies. The proposed protocol with dynamic refreshing achieves the optimal DMT bound in the low multiplexing gain regime and shows the best DMT performance among existing protocols without decoding delay regardless of multiplexing gain. The gain of the proposed protocol in terms of DMT results from both dynamic refreshing and adaptive selection of SIC and joint decoding at each relay.

\section{APPENDIX A \\ PROOF OF THEOREM 1}

Throughout Appendix A, we show how and when the dominant scale of each element of the transition matrix converges when dynamic refresh is adopted. The dominant exponential 
scale of each element in the transition matrix depends on the multiplexing gain, $r$. For the three mainly different regions of the multiplexing gain, the dominant scales of the steady state probability vector $\boldsymbol{\pi}$ are derived as follows:

1) $0<r \leq \frac{m}{3}$

Since $d_{m, m}^{\frac{3}{3}}(r)=\min \left\{d_{m, m}(r), d_{m, 2 m}(2 r)\right\}=$ $d_{m, m}(r)$ for $0<r \leq \frac{m}{3}$,

$$
\begin{aligned}
\mathbf{P} & \doteq\left(\mathbf{P}_{0} \cdots \mathbf{P}_{N_{c}} \mathbf{P}_{N_{c}+1} \cdots \mathbf{P}_{K-1} \mathbf{P}_{K}\right), \\
\mathbf{P}^{2} & \doteq\left(\mathbf{P}_{0}^{2} \cdots \mathbf{P}_{N_{c}}^{2} \mathbf{P}_{N_{c}+1}^{2} \cdots \mathbf{P}_{K-1}^{2} \mathbf{P}_{K}^{2}\right),
\end{aligned}
$$

where

$$
\begin{gathered}
\mathbf{P}_{0}=\cdots=\mathbf{P}_{N_{c}} \doteq\left[\rho^{-K d_{m, m}(r)}, \rho^{-(K-1) d_{m, m}(r)}\right. \\
\left.\quad \cdots, \rho^{-d_{m, m}(r)}, \rho^{0}\right]^{T} \\
\mathbf{P}_{N_{c}+1}=\cdots=\mathbf{P}_{K} \doteq\left[\rho^{-(K-1) d_{m, m}(r)}\right. \\
\left.\rho^{-(K-2) d_{m, m}(r)}, \cdots, \rho^{-d_{m, m}(r)}, \rho^{0}, 0\right]^{T} \\
\mathbf{P}_{0}^{2}=\cdots=\mathbf{P}_{N_{c}}^{2} \doteq\left[\rho^{-(K-1) d_{m, m}(r)}\right. \\
\left.\rho^{-(K-2) d_{m, m}(r)}, \cdots, \rho^{-d_{m, m}(r)}, \rho^{0}, \rho^{-K d_{m, m}(r)}\right]^{T} \\
\mathbf{P}_{N_{c}+1}^{2}=\cdots=\mathbf{P}_{K}^{2} \doteq\left[\rho^{-(K-1) d_{m, m}(r)}, \rho^{-(K-2) d_{m, m}(r)}\right. \\
\left.\quad \cdots, \rho^{-d_{m, m}(r)}, \rho^{0}, \rho^{-(K-1) d_{m, m}(r)}\right]^{T}
\end{gathered}
$$

Note that $\mathbf{P}_{0}=\cdots=\mathbf{P}_{N_{c}}$ if the refreshing criterion of the proposed protocol is $N_{c}$ in any multiplexing gain region. In the same way, we can obtain $\mathbf{P}^{3} \doteq \cdots \doteq$ $\lim _{n \rightarrow \infty} \mathbf{P}^{n} \doteq\left(\mathbf{P}_{0}^{n} \cdots \mathbf{P}_{K}^{n}\right)$, where

$$
\begin{gathered}
\mathbf{P}_{0}^{n}=\cdots=\mathbf{P}_{K}^{n} \doteq\left[\rho^{-(K-1) d_{m, m}(r)}, \rho^{-(K-2) d_{m, m}(r)},\right. \\
\left.\cdots, \rho^{-d_{m, m}(r)}, \rho^{0}, \rho^{-(K-1) d_{m, m}(r)}\right]^{T} .
\end{gathered}
$$

Therefore,

$$
\begin{aligned}
\boldsymbol{\pi} & =\lim _{n \rightarrow \infty} \mathbf{P}_{i}^{n} \\
& \doteq\left[\rho^{-(K-1) d_{m, m}(r)}, \rho^{-(K-2) d_{m, m}(r)}, \rho^{-(K-3) d_{m, m}(r)}\right. \\
& \left.\cdots, \rho^{0}, \rho^{-(K-1) d_{m, m}(r)}\right]^{T} \\
& \triangleq \tilde{\boldsymbol{\pi}}
\end{aligned}
$$

where $\mathbf{P}_{i}^{n}$ is the $(i+1)$-th column of the matrix $\mathbf{P}^{n}$. Since $\rho^{0}$ is only in the $K$-th element of $\tilde{\boldsymbol{\pi}}$,

$$
\begin{aligned}
\lim _{\rho \rightarrow \infty} \boldsymbol{\pi} & \doteq\left[\pi_{0}^{(1)}, \pi_{1}^{(1)} \cdots, \pi_{K-1}^{(1)}, \pi_{K}^{(1)}\right]^{T} \\
& \approx\left[\begin{array}{llllll}
0 & 0 & \cdots & 0 & 1 & 0
\end{array}\right]^{T} .
\end{aligned}
$$

That is, $\lim _{\rho \rightarrow \infty} \pi_{K-1} \approx 1$ and $\lim _{\rho \rightarrow \infty} \pi_{t} \approx 0, \forall t \neq$ $K-1$ where $\pi_{t}$ is the steady state probability of state $t$ corresponding to $(t+1)$-th element of $\boldsymbol{\pi}$. Note that two limits about $n$ and $\rho$ are independent so that the order of limits is irrelevant to the DMT results throughout this paper.

2) $\frac{m}{3}<r \leq \frac{m}{2}$

Since $\bar{d}_{m, m}^{J}(r)=\min \left\{d_{m, m}(r), d_{m, 2 m}(2 r)\right\}=$ $d_{m, 2 m}(2 r)$, which means $d_{m, m}^{J D}(r)<d_{m, m}(r)$, for $r \in\left[\frac{m}{3}, \frac{m}{2}\right], \mathbf{P} \doteq\left(\mathbf{P}_{0} \cdots \mathbf{P}_{N_{c}} \mathbf{P}_{N_{c}+1} \cdots \mathbf{P}_{K-1} \mathbf{P}_{K}\right)$ where

$$
\begin{gathered}
\mathbf{P}_{0} \doteq\left[\rho^{-K d_{m, m}(r)}, \rho^{-(K-1) d_{m, m}(r)}, \rho^{-(K-2) d_{m, m}(r)},\right. \\
\left.\quad \cdots, \rho^{-2 d_{m, m}(r)}, \rho^{-d_{m, m}(r)}, \rho^{0}\right]^{T}, \\
\vdots \\
\mathbf{P}_{N_{c}} \doteq\left[\rho^{-K d_{m, m}(r)}, \rho^{-(K-1) d_{m, m}(r)}, \rho^{-(K-2) d_{m, m}(r)},\right. \\
\left.\quad \ldots, \rho^{-2 d_{m, m}(r)}, \rho^{-d_{m, m}(r)}, \rho^{0}\right]^{T}, \\
\mathbf{P}_{N_{c}+1} \doteq\left[\rho^{-\left\{N_{c} d_{m, m}(r)+\left(K-N_{c}-1\right) d_{m, m}^{J D}(r)\right\}},\right. \\
\rho^{-\left\{\left(N_{c}-1\right) d_{m, m}(r)+\left(K-N_{c}-1\right) d_{m, m}^{J D}(r)\right\}}, \\
\quad \cdots, \rho^{-\left\{d_{m, m}(r)+\left(K-N_{c}-1\right) d_{m, m}^{J D}(r)\right\}}, \\
\rho^{-\left(K-N_{c}-1\right) d_{m, m}^{J D}(r)}, \rho^{-\left(K-N_{c}-2\right) d_{m, m}^{J D}(r)}, \\
\left.\quad \ldots, \rho^{0}, 0\right]^{T}, \\
\quad \vdots \\
\mathbf{P}_{i} \doteq\left[\rho^{-\left\{(i-1) d_{m, m}(r)+(K-i) d_{m, m}^{J D}(r)\right\}},\right. \\
\rho^{-\left\{(i-2) d_{m, m}(r)+(K-i) d_{m, m}^{J D}(r)\right\}}, \cdots, \\
\rho^{-\left\{(K-i) d_{m, m}^{J D}(r)\right\}}, \rho^{-\left\{(K-i-1) d_{m, m}^{J D}(r)\right\}}, \cdots, \\
\left.\rho^{-d_{m, m}^{J D}(r)} \rho^{0}, 0\right]^{T}, \\
\quad \vdots \\
\mathbf{P}_{K-1} \doteq\left[\rho^{-\left\{(K-2) d_{m, m}(r)+d_{m, m}^{J D}(r)\right\}},\right. \\
\left.\rho^{-\left\{(K-3) d_{m, m}(r)+d_{m, m}^{J D}(r)\right\}}, \cdots, \rho^{-d_{m, m}^{J D}(r)}, \rho^{0}, 0\right]^{T},
\end{gathered}
$$$$
\mathbf{P}_{K} \doteq\left[\rho^{-(K-1) d_{m, m}(r)}, \rho^{-(K-2) d_{m, m}(r)},\right.
$$$$
\left.\rho^{-(K-3) d_{m, m}(r)}, \cdots, \rho^{-d_{m, m}(r)}, \rho^{0}, 0\right]^{T},
$$

where $i>N_{c}$. Interestingly, the dominant scales of $\mathbf{P}^{n}$ are different depending on $n, K$, and multiplexing gain, $r$. Each element in the transition matrix is obtained as

$$
P_{i, j}^{k+1}=\sum_{l=0}^{K} P_{l, j}^{k} P_{i, l} \doteq \max _{l} P_{l, j}^{k} P_{i, l}, \forall k \geq 1,
$$

and the scale of $P_{i, j}^{2}$ in $\mathbf{P}^{2}$ is different for two cases: $d_{m, m}(r) \leq 2 d_{m, m}^{J D}(r)$ or not. It should be noted that $P_{i, j}$ is the transition probability from the state $i$ to the state $j$, and corresponds to the $(j+1, i+1)$ entry in the transition matrix $\mathbf{P}$.

- For $d_{m, m}(r) \leq 2 d_{m, m}^{J D}(r)$,

$$
\begin{aligned}
P_{i, j}^{k+1} & =\sum_{l=0}^{K} P_{l, j}^{k} P_{i, l}^{k} \doteq \max _{l} P_{l, j}^{k} P_{i, l}^{k} \\
& =P_{K-1, j}^{k} P_{i, K-1}^{k}=P_{K-1, j}^{k}, \quad \forall k \geq 1
\end{aligned}
$$

where $1+N_{c} \leq i \leq K$ and $0 \leq j \leq K-1$. Let $\boldsymbol{\pi}^{(1)}$ be the dominant scale of the steady state probability 
vector when $d_{m, m}(r) \leq 2 d_{m, m}^{J D}(r)$. After some matrix computation, we can straightforwardly confirm that

$$
\begin{aligned}
\boldsymbol{\pi}^{(1)} & =\lim _{n \rightarrow \infty} \mathbf{P}_{i}^{n}=\left[\pi_{0}^{(1)}, \pi_{1}^{(1)} \cdots, \pi_{K-1}^{(1)}, \pi_{K}^{(1)}\right]^{T} \\
\doteq & \left.\mathbf{P}_{K-1}(1), \mathbf{P}_{K-1}(2), \cdots, \mathbf{P}_{K-1}(K), \pi_{K}^{(1)}\right]^{T}
\end{aligned}
$$

where $\pi_{i}^{(j)}$ is the steady state probability of the $i$-th state in $\boldsymbol{\pi}^{(j)}$ (or the $(i+1)$-th element of $\boldsymbol{\pi}^{(j)}$ ) and $\mathbf{P}_{K-1}(l)$ is the $l$-th element of the vector $\mathbf{P}_{K-1}$. On the other hand,

$$
\begin{aligned}
\pi_{K}^{(1)} & =\lim _{n \rightarrow \infty} P_{K-1, K}^{n}=\lim _{k \rightarrow \infty} \sum_{l=0}^{K} P_{l, K}^{k} P_{K-1, l} \\
& \doteq \lim _{k \rightarrow \infty} P_{0, K} P_{K-1,0} P_{K-1, K-1}^{k} \\
& =P_{0, K} P_{K-1,0} .
\end{aligned}
$$

Therefore, we can finally obtain the dominant scales of the steady state probability vector in $d_{m, m}(r) \leq$ $2 d_{m, m}^{J D}(r)$ given by

$$
\begin{aligned}
\boldsymbol{\pi}^{(1)} \doteq & {\left[\rho^{-\left\{(K-2) d_{m, m}(r)+d_{m, m}^{J D}(r)\right\}},\right.} \\
& \rho^{-\left\{(K-3) d_{m, m}(r)+d_{m, m}^{J D}(r)\right\}}, \cdots, \rho^{-d_{m, m}^{J D}(r)} \\
& \left.\rho^{0}, \rho^{-\left\{(K-2) d_{m, m}(r)+d_{m, m}^{J D}(r)\right\}}\right]^{T} \\
\triangleq & \tilde{\boldsymbol{\pi}}^{(1)}
\end{aligned}
$$

- For $2 d_{m, m}^{J D}(r)<d_{m, m}(r)$, $\mathbf{P}^{2} \doteq\left(\mathbf{P}_{0}^{2} \cdots \mathbf{P}_{N_{c}}^{2} \mathbf{P}_{N_{c}+1}^{2} \cdots \mathbf{P}_{K-1}^{2} \mathbf{P}_{K}^{2}\right)$ where

$$
\begin{gathered}
\mathbf{P}_{0}^{2} \doteq\left[\rho^{-(K-1) d_{m, m}(r)}, \rho^{-(K-2) d_{m, m}(r)},\right. \\
\left.\cdots, \rho^{-d_{m, m}(r)}, \rho^{0}, \rho^{-K d_{m, m}(r)}\right]^{T}, \\
\vdots \\
\mathbf{P}_{N_{c}}^{2} \doteq\left[\rho^{-(K-1) d_{m, m}(r)}, \rho^{-(K-2) d_{m, m}(r)},\right. \\
\left.\cdots, \rho^{-d_{m, m}(r)}, \rho^{0}, \rho^{-K d_{m, m}(r)}\right]^{T},
\end{gathered}
$$

$$
\begin{aligned}
\mathbf{P}_{N_{c}+1}^{2} \doteq\left[\rho^{-\left\{\left(N_{c}-1\right) d_{m, m}(r)+\left(2 K-2 N_{c}-1\right) d_{m, m}^{J D}(r)\right\}},\right. \\
\\
\quad \rho^{-\left\{\left(N_{c}-2\right) d_{m, m}(r)+\left(2 K-2 N_{c}-1\right) d_{m, m}^{J D}(r)\right\}}, \cdots, \\
\quad \rho^{-\left\{d_{m, m}(r)+\left(2 K-2 N_{c}-1\right) d_{m, m}^{J D}(r)\right\}}, \\
\quad \rho^{-\left(2 K-2 N_{c}-1\right) d_{m, m}^{J D}(r)}, \rho^{-\left(2 K-2 N_{c}-3\right) d_{m, m}^{J D}(r)}, \cdots, \\
\left.\quad \rho^{-d_{m, m}^{J D}(r)}, \rho^{0}, \rho^{-\left\{\left(N_{c}-1\right) d_{m, m}(r)+\left(2 K-2 N_{c}-1\right) d_{m, m}^{J D}(r)\right\}}\right]^{T},
\end{aligned}
$$

$$
\begin{aligned}
\mathbf{P}_{i}^{2} \doteq\left[\rho^{-\left\{(i-2) d_{m, m}(r)+(2 K-2 i+1) d_{m, m}^{J D}(r)\right\}},\right. \\
\quad \rho^{-\left\{(i-3) d_{m, m}(r)+(2 K-2 i+1) d_{m, m}^{J D}(r)\right\}}, \\
\quad \cdots, \rho^{-\left\{(2 K-2 i+1) d_{m, m}^{J D}(r)\right\}}, \rho^{-\left\{(2 K-2 i-1) d_{m, m}^{J D}(r)\right\}}, \\
\left.\quad \cdots, \rho^{-d_{m, m}^{J D}(r)}, \rho^{0}, \rho^{-\left\{(i-2) d_{m, m}(r)+(2 K-2 i+1) d_{m, m}^{J D}(r)\right\}}\right]^{T},
\end{aligned}
$$

$$
\begin{aligned}
& \mathbf{P}_{K-1}^{2} \doteq\left[\rho^{-\left\{(K-3) d_{m, m}(r)+3 d_{m, m}^{J D}(r)\right\}}\right. \\
& \rho^{-\left\{(K-2) d_{m, m}(r)+3 d_{m, m}^{J D}(r)\right\}}, \cdots, \\
& \rho^{-\left\{d_{m, m}(r)+3 d_{m, m}^{J D}(r)\right\}} \rho^{-3 d_{m, m}^{J D}(r)}, \rho^{-d_{m, m}^{J D}(r)}, \rho^{0}, \\
&\left.\rho^{-\left\{(K-3) d_{m, m}(r)+3 d_{m, m}^{J D}(r)\right\}}\right]^{T} \\
& \mathbf{P}_{K}^{2} \doteq\left[\rho^{-(K-1) d_{m, m}(r)}, \rho^{-(K-2) d_{m, m}(r)}\right. \\
& \rho^{-(K-3) d_{m, m}(r)}, \cdots, \rho^{-d_{m, m}(r)}, \rho^{0} \\
&\left.\rho^{-(K-1) d_{m, m}(r)}\right]^{T},
\end{aligned}
$$

where $i>N_{c}>0 . \mathbf{P}^{k}(k \geq 3)$ has different dominant scales according to the multiplexing gain regions, $i d_{m, m}^{J D}(r)<d_{m, m}(r) \leq(i+1) d_{m, m}^{J D}(r)$ for $i=2,3, \cdots$. First, for $2 d_{m, m}^{J D}(r)<d_{m, m}(r) \leq$ $3 d_{m, m}^{J D}(r)$

$$
\begin{aligned}
P_{i, j}^{k+1} & =\sum_{l=0}^{K} P_{l, j}^{k} P_{i, l}^{k} \doteq \max _{l} P_{l, j}^{k} P_{i, l}^{k} \\
& =P_{K-1, j}^{k} P_{i, K-1}^{k}=P_{K-1, j}^{k} \forall k \geq 2
\end{aligned}
$$

where $2+N_{c} \leq i \leq K$ and $0 \leq j \leq K-1$. Let $\boldsymbol{\pi}^{(2)}$ be the dominant scale of the steady state probability vector for $\mathbf{P}_{i}^{n}$ in the region, $2 d_{m, m}^{J D}(r)<$ $d_{m, m}(r) \leq 3 d_{m, m}^{J D}(r)$. Similarly to $\boldsymbol{\pi}^{(1)}, \boldsymbol{\pi}^{(2)}$ is obtained as

$$
\begin{aligned}
\boldsymbol{\pi}^{(2)}= & \lim _{n \rightarrow \infty} \mathbf{P}_{i}^{n} \doteq \mathbf{P}_{K-1}^{2} \\
= & {\left[\rho^{-\left\{(K-3) d_{m, m}(r)+3 d_{m, m}^{J D}(r)\right\}},\right.} \\
& \rho^{-\left\{(K-1) d_{m, m}(r)+3 d_{m, m}^{J D}(r)\right\}}, \cdots, \\
& \rho^{-\left\{d_{m, m}(r)+3 d_{m, m}^{J D}(r)\right\}}, \rho^{-3 d_{m, m}^{J D}(r)}, \\
& \left.\rho^{-d_{m, m}^{J D}(r)}, \rho^{0}, \rho^{-\left\{(K-3) d_{m, m}(r)+3 d_{m, m}^{J D}(r)\right\}}\right]^{T} \\
\triangleq & \tilde{\boldsymbol{\pi}}^{(2)}
\end{aligned}
$$

In the same way of computing $\boldsymbol{\pi}^{(1)}$ and $\boldsymbol{\pi}^{(2)}$, we can obtain the general $\boldsymbol{\pi}^{(i)}$, which is the dominant scale of 
the steady state probability vector when $i d_{m, m}^{J D}(r)<$ $d_{m, m}(r) \leq(i+1) d_{m, m}^{J D}(r)$, by

$$
\begin{aligned}
\boldsymbol{\pi}^{(i)}= & \lim _{n \rightarrow \infty} \mathbf{P}_{i}^{n} \doteq \mathbf{P}_{K-1}^{i} \\
= & {\left[\rho^{-\left\{(K-i-1) d_{m, m}(r)+\frac{i^{2}+i}{2} d_{m, m}^{J D}(r)\right\}},\right.} \\
& \rho^{-\left\{(K-i-2) d_{m, m}(r)+\frac{i^{2}+i}{2} d_{m, m}^{J D}(r)\right\}}, \cdots, \\
& \rho^{-\left\{\frac{i^{2}+i}{2} d_{m, m}^{J D}(r)\right\}}, r h o^{-6 d_{m, m}^{J D}(r)}, \rho^{-3 d_{m, m}^{J D}(r)}, \\
& \left.\rho^{-d_{m, m}^{J D}(r)}, \rho^{0}, \rho^{-\left\{(K-i-1) d_{m, m}(r)+\frac{i^{2}+i}{2} d_{m, m}^{J D}(r)\right\}}\right]^{T} \\
\triangleq & \tilde{\boldsymbol{\pi}}^{(i)} .
\end{aligned}
$$

Therefore, there are $K-N_{c}-1$ multiplexing regions for $r \in\left(\frac{m}{3}, \leq \frac{m}{2}\right]$, which lead different dominant scales for the steady state probability vector. For $r \in\left(\frac{m}{3}, \leq \frac{m}{2}\right]$, we can obtain the dominant scale of the steady state probability vector for $r \in\left(\frac{m}{3}, \leq \frac{m}{2}\right]$ when dynamic refreshing is applied as Eq. (60) on the top of the next page.

Since $\rho^{0}$ is only in the $K$-th element of $\tilde{\boldsymbol{\pi}}$,

$$
\begin{aligned}
\lim _{\rho \rightarrow \infty} \boldsymbol{\pi} & =\left[\pi_{0}^{(1)}, \pi_{1}^{(1)} \cdots, \pi_{K-1}^{(1)}, \pi_{K}^{(1)}\right]^{T} \\
& \approx\left[\begin{array}{llllll}
0 & 0 & \cdots & 0 & 1 & 0
\end{array}\right]^{T} .
\end{aligned}
$$

That is, $\lim _{\rho \rightarrow \infty} \pi_{K-1} \approx 1$ and $\lim _{\rho \rightarrow \infty} \pi_{t} \approx 0, \forall t \neq$ $K-1$.

3) $\frac{m}{2}<r \leq m$

Since $\bar{d}_{m, m}^{J D}(r)=0$ for $r \in\left(\frac{m}{2}, m\right], \mathbf{P} \doteq$ $\left(\mathbf{P}_{0} \cdots \mathbf{P}_{N_{c}} \mathbf{P}_{N_{c}+1} \cdots \mathbf{P}_{K-1} \mathbf{P}_{K}\right)$ where

$$
\begin{aligned}
\mathbf{P}_{0} & =\cdots=\mathbf{P}_{N_{c}} \\
& \doteq\left[\rho^{-K d_{m, m}(r)}, \rho^{-(K-1) d_{m, m}(r)}, \cdots, \rho^{-d_{m, m}(r)}, \rho^{0}\right]^{T},
\end{aligned}
$$$$
\mathbf{P}_{i} \doteq\left[\rho^{-(i-1) d_{m, m}(r)}, \rho^{-(i-2) d_{m, m}(r)}, \cdots, \rho^{-d_{m, m}(r)},\right.
$$$$
\left.\rho^{0}, 0\right]^{T},
$$

where $N_{c}+1 \leq i \leq K$. In the same way as in the other multiplexing regions, the dominant scales of the steady state probabilities are obtained by

$$
\begin{aligned}
& \boldsymbol{\pi}= \lim _{n \rightarrow \infty} \mathbf{P}_{i}^{n} \\
& \doteq\left[\rho^{-N_{c} d_{m, m}(r)}, \rho^{-\left(N_{c}-1\right) d_{m, m}(r)}, \cdots, \rho^{-d_{m, m}(r)}, \rho^{0},\right. \\
&\left.\cdots, \rho^{0}, \rho^{0}\right]^{T} \triangleq \tilde{\boldsymbol{\pi}} .
\end{aligned}
$$

Since $\rho^{0}$ appears in the last $K-N_{c}+1$ elements,

$$
\begin{aligned}
\lim _{\rho \rightarrow \infty} \boldsymbol{\pi} & =\left[\begin{array}{lllll}
\pi_{0}, \pi_{1} & \cdots, \pi_{K-1}, \pi_{K}
\end{array}\right]^{T} \\
& \approx\left[\begin{array}{lllllll}
0 & 0 & \cdots & c_{N_{c}} & \cdots & c_{K-1} & c_{K}
\end{array}\right]^{T}
\end{aligned}
$$

and it is easily shown that all the coefficients of $\rho^{0}$ are the same. Therefore, $c_{N_{c}}=\cdots=c_{K}=\frac{1}{K-N_{c}+1}$ and the maximum multiplexing gain when $r \in\left(\frac{m}{2}, m\right]$ is $\frac{K-N_{c}}{K-N_{c}+1}$ because dynamic refreshing occurs when the cardinality of the decoding set is $N_{c}$.

Substituting $\tilde{\pi}_{t}, t=0, \cdots, K$ into Eq. (17), we obtain Eq. (23).

\section{REFERENCES}

[1] J. N. Laneman, D. N. C. Tse, and G. W. Wornell, "Cooperative diversity in wireless networks: efficient protocols and outage behavior," IEEE Trans. Inf. Theory, vol. 50, no. 12, pp. 3062-3080, Dec. 2004.

[2] L. Zheng and D. N. C Tse, "Diversity and multiplexing: a fundamental tradeoff in multiple-antenna channels," IEEE Trans. Inf. Theory, vol. 49, no. 5, pp. 1073-1096, May 2003.

[3] R. U. Nabar, H. Bolcskei, and F. W. Kneubuhler, "Fading relay channels: performance limits and space=time signal design," IEEE J. Sel. Areas Commun., vol. 22, no. 6, pp. 1099-1109, Aug. 2004.

[4] K. Azarian, H. El Gamal, and P. Schniter, "On the achievable diversitymultiplexing tradeoff in half-duplex cooperative channels," IEEE Trans. Inf. Theory, vol. 51, no. 12, pp. 4152-4172, Dec. 2005.

[5] P. Mitran, H. Ochiai, and V. Tarokh, "Space-time diversity enhancements using collaborative communications," IEEE. Trans. Inf. Theory, vol. 51, no. 6, pp. 2041-2057, June 2005.

[6] M. Katz and S. Shamai (Shitz), "Transmitting to colocated users in wireless adhoc sensor networks," IEEE. Trans. Inf. Theory, vol. 51, no. 10, pp. 3540-3563, Oct. 2005 .

[7] M. Yuksel and E. Erkip, "Multi-antenna cooperative wireless systems: a diversity-multiplexing tradeoff perspective," IEEE. Trans. Inf. Theory, vol. 53, no. 10, pp. 3371-3393, Dec. 2007.

[8] S. Pawar, A. S. Avestimehr, and D. N. C. Tse, "Diversity-multiplexing tradeoff of the half-duplex relay channel," in Proc. 2008 Allerton Conf. Communication, Contro, and Computing, pp. 27-33.

[9] B. Escrig, "DMT optimal cooperative protocol with destination-based selection of the best relay," IEEE Trans. Wireless Commun., vol. 10, no. 7, pp. 2218-2227, July 2011.

[10] H. E. Gamal, G. Caire, and M. O. Damen, "The MIMO ARQ channel: diversity-multiplexing-delay tradeoff," IEEE Trans. Inf. Theory, vol. 52, no. 8, pp. 3601-3621, Aug. 2006.

[11] B. Rankov and A. Wittneben, "Spectral efficeint protocols for halfduplex fading relay channels," IEEE J. Sel. Areas Commun., vol. 25, no. 2, pp. 379-389, Feb. 2007.

[12] Y. Fan, C. Wang, J. Thompson, and H. V. Poor, "Recovering multiplexing loss through successive relaying using repetition coding," IEEE Trans. Wireless Commun., vol. 6, no. 12, pp. 4484-4493, Dec. 2007.

[13] A. Bletsas and A. G. Dimitriou, "Interference-limited relaying with reactive sensing," IEEE Trans. Wireless Commun., vol. 9, no. 1, pp. 1420, Jan. 2010.

[14] S. Yang and J. C. Belfiore, "Towards the optimal amplify-and-forward cooperative diversity scheme," IEEE. Trans. Inf. Theory, vol. 53, no. 9, pp. 3114-3126, Sept. 2007.

[15] S. O. Gharan, A. Bayesteh, and K. Khandani, "On the diversitymultiplexing tradeoff in multiple-relay network," IEEE. Trans. Inf. Theory, vol. 55, no. 12, pp. 5423-5444, Dec. 2009.

[16] B. Hong and W. Choi, "Overcoming half-duplex loss in multi-relay networks: multiple relay coded cooperation for optimal DMT," submitted to IEEE. Trans. Wireless Commun..

[17] R. Tannious and A. Nosratinia, "Spectrally-efficient relay selection with limited feedback," IEEE J. Sel. Areas Commun., vol. 26, no. 8, pp. 14191428, Oct. 2008.

[18] Y.-B. Kim and W. Choi, "Interference cancelation based opportunistic relaying with multiple decode-and-forward relays," 2010 IEEE Veh. Tech. Conf..

[19] J. Ryu and W. Choi, "Bit concatenation based user relaying in MIMO broadcast channels," IEEE Trans. Commun., vol. 60, no. 8, pp. 22082220, Aug. 2012.

[20] J. Ryu, W. Choi, and D. I. Kim, "Partial stream relaying in MIMO relay communications," IEEE Trans. Veh. Technol., vol. 62, no. 1, pp. 205-218, Jan. 2013.

[21] A. Bletsas, A. Khisti, D. P. Reed, and A. Lippman, "A simple cooperative diversity method based on network path selection," IEEE J. Sel. Areas Commun., vol. 24, no. 3, pp. 659-672, Mar. 2006.

[22] S. M. Ross, Stochastic Process, 2nd ed. John Wiley \& Sons, Inc., 1996.

[23] M. Ansari, A. Bayesteh, and A. K. Khandani, "Diversity-multiplexing tradeoff in Z-channel," in Proc. 2007 Canadian Workshop on Information Theory, pp. 21-24.

[24] S. Karmakar and M. K. Varanasi, "The diversity-multiplexing tradeoff of the MIMO Z interference channel," July 2010, preprint. Available: http://www.arxiv.org 


$$
\boldsymbol{\pi}=\left\{\begin{array}{ccc}
\boldsymbol{\pi}^{(1)}, & \text { if } & d_{m, m}(r) \leq 2 d_{m, m}^{J D}(r) \\
\boldsymbol{\pi}^{(2)}, & \text { if } & 2 d_{m, m}^{J D}(r)<d_{m, m}(r) \leq 3 d_{m, m}^{J D}(r) \\
\vdots & & \\
\boldsymbol{\pi}^{(i)}, & \text { if } & i d_{m, m}^{J D}(r)<d_{m, m}(r) \leq(i+1) d_{m, m}^{J D}(r) \\
\vdots & & \\
\boldsymbol{\pi}^{\left(K-N_{c}-2\right)}, & \text { if } & \left(K-N_{c}-2\right) d_{m, m}^{J D}(r)<d_{m, m}(r) \leq\left(K-N_{c}-1\right) d_{m, m}^{J D}(r) \\
\boldsymbol{\pi}^{\left(K-N_{c}-1\right)}, \text { if } & \left(K-N_{c}-1\right) d_{m, m}^{J D}(r)<d_{m, m}(r)
\end{array} .\right.
$$

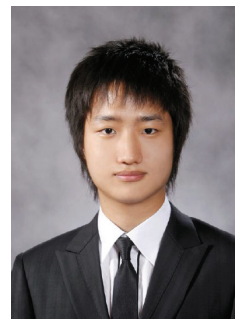

Young-bin Kim (S'11) received the B.S. degrees in Electrical and Computer Engineering from Information and Communications University (ICU), which merged into Korea Advanced Institute of Science and Technology (KAIST) on March 1 in 2009, Korea, in 2008. He received the M.S. degrees in Electrical Engineering from KAIST in 2010. $\mathrm{He}$ is currently pursuing the Ph.D. degree in the Department of Electrical Engineering from KAIST. His research interests include cooperative communication, interference management, complex network and physical-layer security in communication theory and information theory.

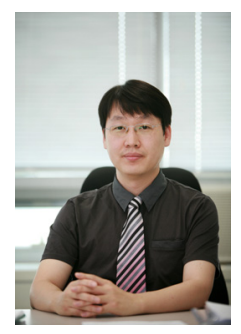

Wan Choi (S'03-M'06-SM12) received the B.Sc. and M.Sc. degrees from the School of Electrical Engineering and Computer Science (EECS), Seoul National University (SNU), Seoul, Korea, in 1996 and 1998, respectively, and the Ph.D. degree in the Department of Electrical and Computer Engineering at the University of Texas at Austin in 2006. He is currently an Associate Professor of the Department of Electrical Engineering, Korea Advance Institute of Science and Technology (KAIST), Daejeon, Korea. From 1998 to 2003, he was a Senior Member of the Technical Staff of the R\&D Division of KT Freetel Company, Limited, Korea. Dr. Choi is the recipient of IEEE Vehicular Technology Society Jack Neubauer Memorial Award which recognized the best paper published in the IEEE Transactions on Vehicular Technology for 2001. He also received the IEEE Vehicular Technology Society Dan Noble Fellowship Award in 2006 and the IEEE Communication Society Asia Pacific Young Researcher Award in 2007. While at the University of Texas at Austin, he was the recipient of William S. Livingston Graduate Fellowship and Information and Telecommunication Fellowship from Ministry of Information and Communication (MIC), Korea. He serves as Associate Editor for the IEEE TRANSACTIONS ON WiRELESS COMMUNiCATIONS, for the IEEE TRANSACTIONS ON VEHICULAR TECHNOLOGY, and for IEEE WIRELESS COMMUNICATIONS LETTERS.

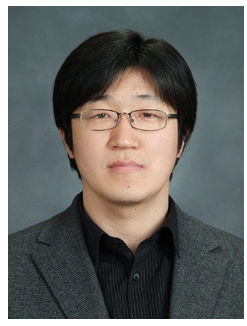

Bang Chul Jung (S'02-M'08) received the B.S. degree in Electronics Engineering from Ajou University, Suwon, Korea, in 2002 and the M.S. and Ph.D degrees in Electrical \& Computer Engineering from KAIST, Daejeon, Korea, in 2004 and 2008, respectively. He was a senior researcher/research professor with KAIST Institute for Information Technology Convergence, Daejeon, Korea, from January 2009 to February 2010. He is now an assistant professor of Department of Information and Communication Engineering, Gyeonsang National University, Korea. Dr. Jung was the recipient of the Fifth IEEE Communication Society AsiaPacific Outstanding Young Researcher Award in 2011. His research interests include wireless communications, statistical signal processing, compressed sensing, information theory, etc.

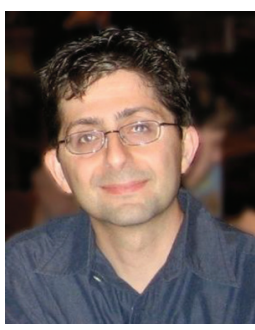

Aria Nosratinia (S'87-M'97-SM'04-F'10) is Jonsson Distinguished Professor of Engineering at the University of Texas at Dallas. He received his $\mathrm{Ph} . \mathrm{D}$. in Electrical and Computer Engineering from the University of Illinois at Urbana-Champaign in 1996. He has held visiting appointments at Princeton University, Rice University, and UCLA. His interests lie in the broad area of information theory and signal processing, with applications in wireless communications. He was the secretary of the IEEE Information Theory Society in 2010-2011 and the treasurer for ISIT 2010 in Austin, Texas. He has served as editor for the IEEE TRANSACTIONS ON INFORMATION THEORY, IEEE TRANSACTIONS on Wireless Communications, IEEE Signal Processing LetTers, IEEE Transactions on Image Processing, and IEEE Wireless Communications (Magazine). He has been the recipient of the National Science Foundation career award, and is a fellow of IEEE. 